\title{
Détermination expérimentale des coefficients de transmission de chaleur dans le cas de densités de flux de chaleur élevées dans un milieu à une et à deux phases
}

\author{
Experimental determination \\ of the heat transfer coefficients \\ at high heat flux densities in single-and two-phase media
}

PAR M. BUTZBACH

JNGENIEUR A IA SOCIÉTE ALSTHOM A BELFORT

ANCIEN LLÈVE DE L'ÉCOLE POLYTECHNIQUE

\begin{abstract}
«Les fux de chaleur élevés dégagés dans les réacteurs nucléaires ont imposé des études expérimentales de transmission de chalcur dans l'eau en convection forcée avec on sans ébullition de surface. Les résultats obtenus par les Américains dans ce domaine sont rappelés. L'installation d'essais réalisés pollr vérifier les conditions de refroidissement dans les canaux de la pile EL.3 a permis de recouper quelques-uns de ces résultats; dans le cas de la convection forcée sans ébullition, la formule de Colburn peut être utilisée moyennant certains ajustements. Pour l'ébullition de surface avec bulles, seules des formules empiriques approximatives sont proposées. 》
\end{abstract}

Les études expérimentales de transmission de chaleur dans l'eau à densité de flux élevée, qui font l'objet de cet exposé, ont été menées pour le compte du C.E.A. et en liaison étroite avec celui-ci et les Chantiers de l'Atlantique. Ces études ont eu pour but de se rendre compte des conditions exactes dans lesquelles se ferait le refroidissement des barreaux de la pile EL. 3.

Avant d'en examiner les résultats, il est essentiel de rappeler d'abord pour quelles raisons elles ont été effectuées; ensuite nous verrons comment se caractérise qualitativement la transmission de chaleur dans des milieux à une et à deux phases, et à quelles conclusions sont arrivés les Américains à la suite des séries d'expériences exécutées également en vue de mieux connaître les caractéristiques de transfert de chaleur dans les réacteurs nucléaires.

\begin{abstract}
Experimental investigation of forced connection transmission of heat in water with or withont surface boiling has been made necessary by the high heal flures given off in nuclear reaclors. The resulls of American work in this field are recapitulated. Some of these results were cross-checked on the lest rig which was buill to check cooting conditions in the ducts of pile EL.3.

When certain adjustmenls have been made to il, Colburn's formula can be used for forced convection without boiling. OnIy empirical formulae are suggested for use with bubbling surface boiling.
\end{abstract}

I

Pour la construction des appareils ou machines industriels courants, qu'il s'agisse d'échangeurs de chaleur et surtout de générateurs de vapeur, nous avons été habitués à considérer des densités de flux de chaleur de plus en plus élevées au fur et à mesure du développement des techniques qui leur sont propres. Mais même à l'heure actuelle, dans les chaudières marines où les conditions de fonctionnement sont les plus sévères et aux points les plus chauds, les densités de flux de chaleur transmis à travers les tubes d'eau de la chambre de combustion, sont au plus de l'ordre de $400.000 \mathrm{kcal} / \mathrm{h} / \mathrm{m}^{2}$. Ce chiffre correspond à un taux de dégagement de calories dans la chambre de plus de $10^{6} \mathrm{kcal} / \mathrm{h} / \mathrm{m}^{3}$. 


\section{Nomenclature dES SYMBOLES UTILISÉS}

1 désigne une température de métal.

T désigne une température de fluide chauifé.

$t_{p}$ température de paroi du métal côté fluide chauflé.

$\mathrm{T}_{m}$ lempérature de la masse di fluide chauffé.

$\Gamma_{s a t}$ température de saturation du fluide chauffé.

$\Delta \Theta=t_{p}-\mathbf{T}_{w}$.

$\Delta \Theta_{s a t}=t_{p}-\mathrm{T}_{\mathrm{q} a t}$.

$\Delta \mathrm{T}_{s n t}=\mathrm{T}_{s a t}-\mathrm{T}_{m}$.

$\mathrm{T}_{f}=\frac{l_{p}+\mathrm{T}_{m}}{\mathbf{2}}$ température du film.

$V$ vitesse du fluide chanffé.

L longueur du tube chauffant.

$h$ coefficient de transmission de chaleur entre métal et fluide chauffé.

Q densité de flux de chaleur.

$\varphi_{l}$ densilé de flux de chaleur en un point du tube chauffant.

$c_{p} \quad$ chaleur spécifique du fluide chauffé à pression constante.

$\mu \quad$ viscosité ( $\mu_{p}$ viscosité à la paroi du métal; $\mu_{m}$ viscosité de la masse du fluide chauff $\left.\dot{e}\right)$.

- masse spécifique.

$\lambda \quad$ conductibilité du fluide chauffé.

D diamètre intérieur du tube ou diamètre équivalent intervenant dans le nombre de Reynolds.

P pression du fluide chauffé.

Ms $=\frac{h}{c_{p} V_{o}}$ nombre de Stanton ou de Margoulis (ou $M$ ).

$\operatorname{Pr}=\frac{c_{p} y}{\lambda}$ nombre de Prandtl (ou $P$ ).

$\mathrm{Bi}=\frac{h \mathrm{D}}{\lambda}$ nombre de Biot ou de Nusselt (ou $B$ ).

$\operatorname{Re}=\frac{V_{\rho} D}{\mu}$ nombre de Reynolds (ou $R$ ).

Or, dans le domaine des réacteurs nucléaires, les densités de flux de chaleur à évacuer sont

Nous avons mis en relief la nécessité de l'expérimentation pour l'étude des densités de flux de chaleur élevées et l'intérêt présenté par l'eau comme fluide refroidisseur. facilement de $1 \times 10^{6} \mathrm{kcal} / \mathrm{h} / \mathrm{m}^{2}$, soit $116 \mathrm{w} / \mathrm{cm}^{2}$, et peuvent atteindre $10^{7} \mathrm{kcal} / \mathrm{h} / \mathrm{m}^{2}$ (ces ordres de grandeur se rencontrent d'ailleurs dans l'aviation dans certains engins de propulsion).

La comparaison de ces chiffres - le rapport des densités de flux est compris entre 2,5 et 25 suffit à montrer qu'il n'est pas possible, pour construire des réacteurs nucléaires, de recourir à une extrapolation à partir des résultats relatifs aux techniques classiques; l'expérimentation s'avère indispensable aussi bien pour se rendre compte des phénomènes particuliers qui peuvent se produire pour ces valeurs élevées de la densité de flux que pour oblenir des hases de calcul convenables.

Une fois cette constatation faite, il $\mathrm{y}$ a lieu de procéder au choix du fluide refroidisseur le plus approprié, suivant lai densité de flux de chaleur transmise, en tenant compte des impératifs qui peuvent intervenir, soit du point de vue nucléaire, soit $\mathbf{d u}$ point de vue récupération d'énergie, soit enfin du point de vue métallurgique et chimique.

Les considérations d'ordre métallurgique et chimique sont particulièrement importantes, car les métaux ou alliages utilisés pour le gainage $d u$ combustible ne résistent correctement qu'à des températures relativement basses : il y a intérêt à abaisser l'écart de température entre la gaine et la masse du fluide refroidisseur, donc à rechercher ce dernier de telle facon qu'il ait un coefficient de transmission aussi élevé que possible.

Trois fluides refroidisseurs sont ainsi actuellement retenus : le $\mathrm{CO}_{2}$ sous pression, l'eau, le sodium liquide. Une estimation de Glasstone situe bien les possibilités thermiques de ces trois fluides :

Dans le cas d'un écoulement turbulent à $204{ }^{\circ} \mathrm{C}$ dans un tube circulaire de diamètre $30 \mathrm{~mm}$ :

\begin{tabular}{|r|r|r|r|}
\hline & $\begin{array}{r}\mathrm{CO}_{2 .} \text { sous 10 } \\
\text { atmosphères }\end{array}$ & Eau & $\begin{array}{r}\text { Sodium } \\
\text { liquide. }\end{array}$ \\
\hline $\mathrm{V} \mathrm{m} / \mathrm{s} \ldots \ldots \ldots$ & 30 & 6 & 6 \\
$\mathrm{~h} \mathrm{kcal} / \mathrm{h} / \mathrm{m}^{2} \circ / \mathrm{C}$. & 605 & 39800 & 47100 \\
\hline
\end{tabular}

II

Précisons maintenant, pour mieux comprendre cet intérêt, les particularités de la transmission de chaleur dans l'eau, d'une part, et dans le mélange eau et vapeur, c'est-à-dire quand ces 
deux phases sont en présence, d'autre part. Nous définirons au passage la terminologie employée, pour éviter toute équivoque, étant donné le nombre d'appellations rencontrées.

La figure 1, donnée par Mac Adams (1) et JENs (2) représente en coordonnées logarithmiques la variation de la densité de flux de chaleur en fonction de la différence de température en-

Systeme ò flux de chaleur constant

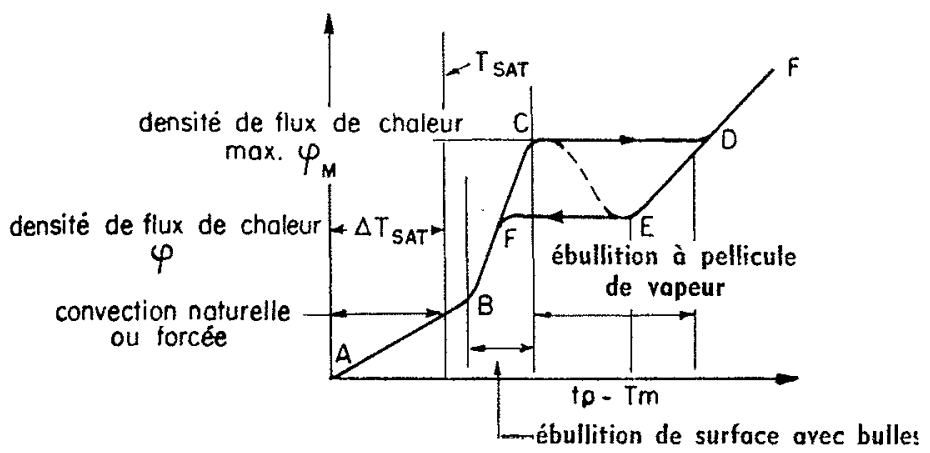

fil, portion DF de la courbe. En opérant à densité de flux décroissante, partant du point $F$ par exemple, l'ébullition à pellicule de vapeur subsiste à des densités intérieures à la densité maximum, mais il peut $\mathrm{y}$ avoir des fonctionnements instables dans la partie $\mathrm{DE}$ de la courbe. Les résultats dans ce cas sont peu sûrs. Seule la courbe $A B C D F$ est bien ćlablie par l'expérience.

\section{Système à température constonte}

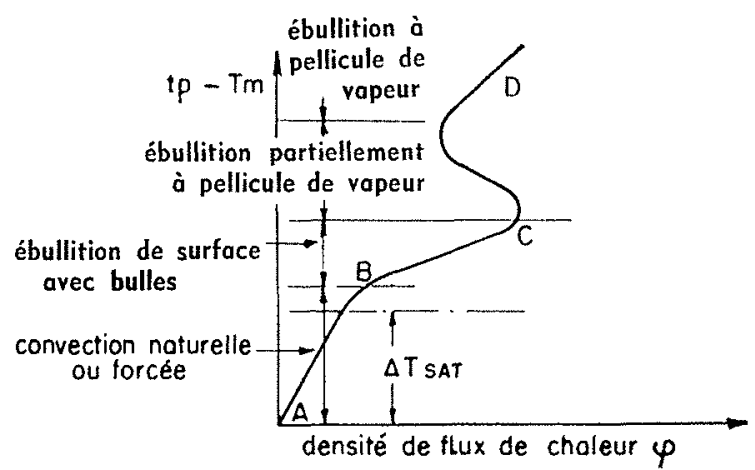

(échelles logarithmiques pour $\varphi$ et tp-Tm)

FIG. 1

tre la surface chauffante et la masse d'eau stagnante, lorsqu'un fil chauffé électriquement traverse cette masse d'eau dont la température est celle de la saturation à la surface. Trois zones sont à distinguer :

- La première correspond à la convection naturelle qui s'établit à faible densité de flux; l'ébullition a lieu seulement à la surface de la masse d'eau.

-- Dans la deuxième, la température de la surface du fil dépasse celle de saturation de l'eau : une ébullition de surface avec formation de bulles se produit (c'est le « nucleate boiling » dans la littérature américaine) que nous désignerons par la suite comme ébullition de surface avec bulles; dans cette zone, la densité de flux de chaleur croît beaucoup plus rapidement que la différence de température, le coefficient de transmission devient très grand.

Mais cet avantage ne subsiste que jusqu'à la densité maximurn de flux de l'ébullition de surface avec bulles, car pour cette densité une augmentation brutale de température $d u$ fil est observée en même temps que l'ébullition change de nature et devient une ébullition avec formation d'une pellicule de vapeur isolant l'eau du fil : c'est la troisième zone. Si le fil peut supporter cette surchauffe instantanée, la densité de flux continue à crô̂tre jusqu'à la fusion du
Dans le cas de la convection forcée, avec de l'cau à une température inférieure à la saturation, une courbe semblable peut être admise, mais on ne peut considérer comme valables que les deux premières zones correspondant cette fois-ci à la convection forcée $\emptyset=h\left(t_{p}-\mathrm{T}_{m}\right)$ et à l'ébullition de surface avec bulles. L'augmentation du coefficient de transmission dans celte zone peut être attribuée à une augmentation de la turbulence de l'eau due à la présence des bulles qui se condensent au contact de la masse du liquide plus froid. Le temps de vie des bulles est très court, 220 microsecondes d'après des photographies de Gunther (3) de la surface d'une barre de mélal chauffée ćlectriquement et disposće dans l'axe d'un canal reclangulaire horizonlal; l'épaisseur de la couche où se produit la condensation des bulles formées sur la paroi chauffante est très faible. Du fait de la convection forcée, les densités de flux transmis sont très élevées et l'on peut arriver au point de «brûlure» du tube (Burnout point) avant d'atteindre la densité maximum de flux.

Lorsque celle-ci est obtenue, la surchaufle du tube se produit instantanément et entraîne sa destruction en un temps extrêmement court. La densité maximum de flux de l'ébullition de surface avec bulles et la densité de flux de brûlure du tube sont ainsi souvent confondues dans les comptes rendus des nombreuses expériences effectuées aux Etats-Unis pour déterminer la valeur du flux maximum. 
Le schéma de transmission de chaleur que nous venons d'éludier correspond à ce qui se passe pour une disposition à flux de chaleur constant, comme c'est le cas des réacteurs nucléaires. Il est à remarquer que la succession des différents modes d'ébullition serait modifiée si l'on considérait une installation à tempéra- ture de paroi constante, comme ce peut être le cas d'échangeur's de chaleur (fig. 1); une fois le point $\mathrm{D}$ atteint, en diminuant l'écart de température, l'ébullition se ferait à certains endroits à pellicule de vapeur à d'autres avec bulles, jusqu'au point $C$ où l'ébullition de surface avec bulles est retrouvée normale.

\section{LES RESULTATS D'ESSAIS AMÉRICAINS}

Ces indications qualitatives sur le processus de transmission de chaleur avec et sans ébullition vont nous permettre d'avoir une vue d'ensemble sur les résultats d'essais obtenus aux Etats-Unis.

a) Nous examinerons d'abord les expériences relatives à la zone de la convection forcée sans ébullition (1) (4): il s'agit d'un domaine connu tout au moins tant que la température de l'eau reste très éloignée de la saturation, mais la prospection s'est étendue à des pressions et des densités de flux de chaleur plus élevées qu'auparavant. La relation classique entre les nombres sans dimension de Stanton, Prandtl et Reynolds, reste toujours valable, $h / c_{p} V_{p}$ étant le nombre de Stanton ou de Margoulis :

$$
\text { Ms } \operatorname{Pr}_{f}^{2 / 3}=0,023 \operatorname{Re}_{f}^{-0,2}
$$

Les caractéristiques physiques du fluide $u, \lambda$, $c_{p}$ pour le nombre de Prandtl et le nombre de Reynolds étant prises à une température conventionnelle dite température du film :

$$
\mathrm{T}_{f}=\frac{t_{p}+\mathrm{T}_{m}}{2} \text { (désignation } \mathrm{Pr}_{f} \text { et } \mathrm{Re}_{f} \text { ) }
$$

Cette validité a été vérifiée jusqu'à des nombres de $\operatorname{Re}$ de $10^{\circ}$ et à des $\Delta \Theta$ élevés :

$$
\left(\Delta \Theta=t_{p}-\mathrm{T}_{m}\right) \text {. }
$$

Les renseignements sur l'influence éventuelle du rapport $L / D$ sont insuffisants, a partir naturellement du point où le régime turbulent est bien établi. La dispersion des résultats paraît, comme dans les conditions habituelles, être inférieure à $\pm 20 \%$ Une autre relation est parfois utilisée :

$$
\mathrm{Ms} \mathrm{Pr}_{f}^{2 / 3}\left(\frac{u_{p}}{\mu_{m}}\right)^{0,14}=0,023 \mathrm{Re}^{-0,2}
$$

Loujours pour tenir compte de la variation de température entre paroi et masse du fluide.

Pour la perte de charge, le coefficient de frottement avec flux de chaleur se déduit du coefficient correspondant à un écoulement isotherme par la relation:

$$
=\frac{f_{\varphi}}{f}\left(\frac{\mu_{p}}{u_{m}}\right)_{(1)(5)}^{0.14}
$$

b) Il est beaucoup plus difficile de rassembler dans un même moule les résultats trouvés sur l'ébullition de surface avec bulles; les paramètres qui entrent en jeu sont très nombreux puisqu'il faut faire intervenir ceux caractérisant chacune des deux phases, c'est-à-dire $y, \lambda, c_{p}$, p pour l'eau et la vapeur, et ceux provenant de la présence de ces deux phases au même point, la tension superficielle de l'eau, la densité du mélange eau-vapeur, la chaleur latente de vaporisation, la température de saturation; d'autres éléments sont également à jetenir comme la teneur en gaz dissous de l'eau, la nature el l'état de la surface chauffante.

En raison de cette complexité du problème, i] n'a pas été trouvé par l'analyse dimensionnelle de relation satisfaisante, même si l'on tient compte des recherches de Rohsenow (6) sur ce point. Aussi les expérimentateurs américains ont cherché des formules aussi simples que possible où figurent seulement les parametres dont l'influence d'après l'expérience est déterminante. L'écart de température entre la paroi et la saturation de l'eau au point considéré :

$$
\Delta \Theta_{s a t}=t_{p}=\mathbf{T}_{a a t}
$$

a été admis comme le plus caractéristique; il a été trouvé que la vitesse de l'eau, l'écart entre la température de saturation et celle de l'eau $\Delta \mathrm{T}_{\text {aat }}=\mathrm{T}_{\varepsilon_{11} t}-\mathrm{T}_{m}$, n'exerçaient qu'une faible influence sur cet écart. lorsque le flux de chaleur croît, l'effet de la vitesse diminue; par contre, sa valeur est essentiellement fonction de la pression. JENs el LooTres (7) donnent l'expression suivante pour traduire ces variations de $\Delta \Theta_{s a t}$ en fonction de $\mathrm{P}$ : 


$$
\Delta \Theta_{s a t}=0,825 \frac{\varphi^{0,25}}{e^{\mathrm{P} / 0,3,1}}
$$

(e en $\mathrm{kcal} / \mathrm{h} / \mathrm{m}^{2}-\mathrm{P}$ en $\mathrm{kg} / \mathrm{cm}^{2}$ )

mais la dispersion est importante, de l'ordre de $\pm 50 \%$ (pressions jusqu'à $175 \mathrm{~kg} / \mathrm{cm}^{2}-0$ $\left.=6,78 \times 10^{6} \mathrm{kcal} / \mathrm{h} / \mathrm{m}^{2}\right)$, ou, ce qui revient au même, à pression déterminée $\varrho=\alpha \Delta \Theta^{4}{ }_{3 a t}$. Mac ADsms indique cette même relation pour l'ébullition de surface avec bulles aussi bien en convection forcée que dans la masse d'un liquide, l'exposant pouvant varier de 3 à 4; dans un espace annulaire, la relation trouvée fut $\varphi=a \Delta \Theta_{s a t^{\natural}, 86}$. L'écart $\Delta \Theta_{s a t}$ est toujours indiqué, à flux donné, comme décroissant avec la pression; la relation proposée par Kreith et SumMERFIELD est par exemple : $\Delta \Theta_{s a t}=a \mathrm{P}^{-3 / 4}$.

Aucune conclusion ne peut être tirée de l'influence de la teneur en gaz dissous dans l'eau, certains la tenant pour négligeable, d'autres estimant qu'elle favorise l'ébullition de surface avec bulles; l'influence de l'état de surface est au contraire bien établie (5): pour deux surfaces de polis différents 1 et $1,25 u$, le rapport des $\Delta \Theta_{s a t}$ est de 0,75 au moins, l'avantage ćtant pour la surface polie: observation paradoxale qui s'explique si l'on admet que la nouillabilité de la surface exerce une influence sur la transmission de chaleur. Cette influence est mise en valeur sur la figure 2 où sont comparées des fluides de mouillabilité différente (8).

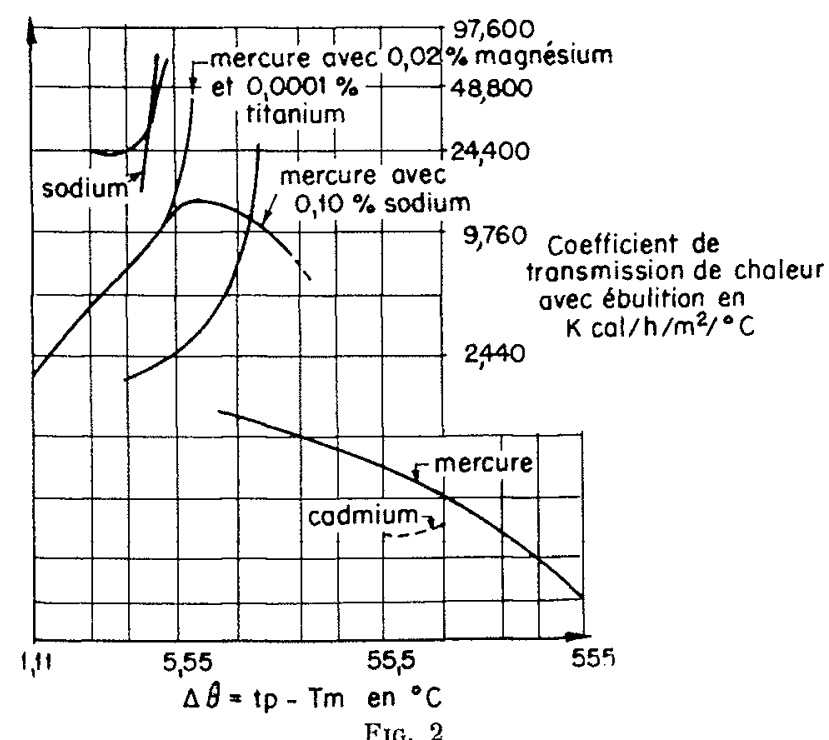

Comparaison des coefficients de transmission de chaleur avec ébullition pour l'eau et les métaux liquides.

Nous ne connaissons que fort peu de résultats sur la valeur de la perte de charge dans les cas où il y a ébullition de surface avec bulles. La figure 3 montre l'augmentation de la perte de charge au fur et à mesure de l'accroissement de la longueur du tube intéressée par l'ébullition de surface. Cette constatation n'est pas étonnante, si nous admettons, comme nous l'avons indiqué auparavant, que l'augmentation du coefficient de transmission est due à une turbulence plus grande causée par la présence des bulles de vapeur.

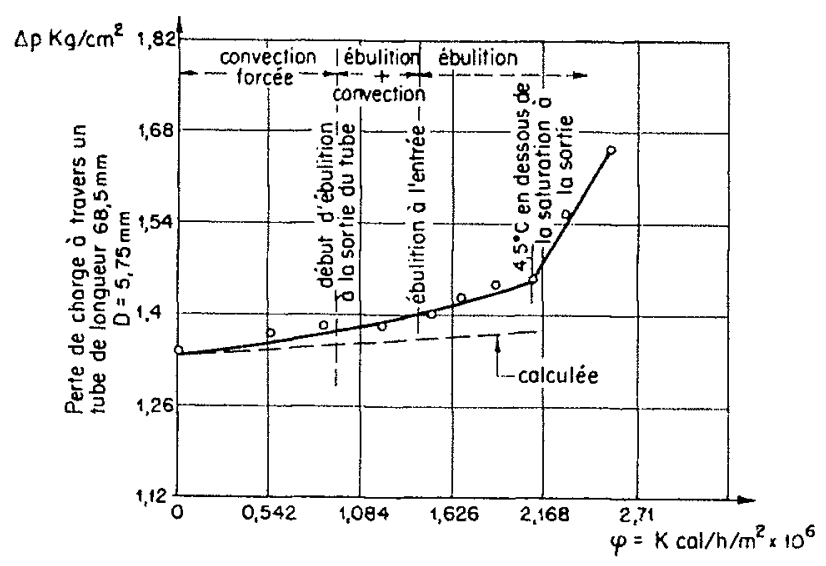

FIG. 3

Comparaison de la perte de charge avec ébullition de surface, avec la perte de charge coleuléc.

Débit : $37,4 \times 10^{\mathrm{B}} \mathrm{kg} / \mathrm{m}^{2} / \mathrm{h}$,

Température d'entrée : $315^{\circ} \mathrm{C}$,

Pression : $140 \mathrm{~kg} / \mathrm{cm}^{2}$.

(Résultats de l'Université de Californie)

c) De nombreux résultats donnent des indications sur les conditions dans lesquelles se produit la brûlure du tube ou sa surchauffe instantanée. Ils permettent en particulier de définir la valeur de la densité de flux maximum de chaleur correspondant, mais les expériences sont difficiles à réaliser car le phénomène est brutal, les caractéristiques du fonctionnement an moment de l'accident repérables souvent tl'is approximativement. Enfin, du fait même de la surchaufe instantanée, il peut $y$ avoir un accroissement brutal de la perte de charge par suite de la formation de nombreuses bulles de vapeur, d'où une diminution du débit d'eau refroidissant le tube : toutes les conditions sont réunies pour aboutir à la brûlure du tube en un temps très court, inférieur à la seconde. Il a été remarqué que de plus grandes valeurs de la densité maximum ox pouvaient etre obtenues en diminuant l'écart $\Delta \mathrm{T}_{\text {rat }}$ ou la vilesse de l'eau au lieu d'accroître le flux de chaleur.

De l'examen des diverses formules proposces reliant la densité oy à la vitesse et à l'écart $\Delta \mathrm{T}_{s i t}$, [abaques avec $\varphi_{\mathrm{M}}=\mathrm{F}\left(\mathrm{V} . \Delta \mathrm{T}_{\text {kat }}\right]$ il ressort, étant donné les divergences constatées, qu'elles ne peuvent être considérées que comme fournissant des ordres de grandeur. Nous ne citerons que les plus classiques : 
(Mac Avams) $\varphi=\left[1,61 \times 10^{6}+3,4 \times 10^{4}\right.$

$$
\left.\times\left(\mathrm{T}_{\text {sat }}-\mathrm{T}_{m}\right)\right] \mathrm{V}^{1 / 3}
$$

( $V$ de 0,3 à $3,6 \mathrm{~m} / \mathrm{s}$ )

( $P$ de 2,1 à $6,3 \mathrm{~kg} / \mathrm{cm}^{2}$ )
(JENS et LOTTES) $\varphi=\alpha\left(\frac{\mathrm{V}_{0}}{10^{6}}\right)^{b}\left(\mathrm{~T}_{s a t}-\mathrm{T}_{m}\right)^{0,22}$

$\left(V\right.$ de 1,5 à $9 \mathrm{~m} / \mathrm{s}-\mathrm{P}$ de 35 à $\left.140 \mathrm{~kg} / \mathrm{cm}^{2}\right)$ les coefficients $a$ et $b$ étant variables suivant la pression (*).
Par rapport au champ d'investigation que nous venons de parcourir et où, comme nous l'avons vu, de nombreux travaux de défrichage ont déjà été entrepris, le domaine des essais que nous avons effectués et que nous effectuons encore maintenant pour la pile EL. 3, est relativement restreint. Le but principal de ces essais était de connaître, pour des conditions de refroidissement déterminées, vitesse et température de l'eau, quels seraient les coefficients de transmission dans un canal de la pile, c'est-à-dire en espace annulaire et par conséquent la température atteinte par la gaine protégeant l'uranium pour une densité de flux de chaleur maximum dégagé à travers la gaine de $200 \mathrm{w} / \mathrm{cm}^{2}$, soit $1,72 \times 10^{6} \mathrm{kcal} / \mathrm{h} / \mathrm{m}^{2}$. Des essais avec les mêmes densités de flux de chaleur devaient être exécutés parallèlement avec de l'eau circulant dans un tube pour permettre des recoupements avec des résultats bien connus en convection forcée sans ébullition; de cette manière, le mode opératoire comme les méthodes de mesure ont pu être contrôlés.

La densité de flux demandée a été obtenue par effet Joule dans un tube chauffé électriquement.

L'installation d'essais (fig. 4) comprend pompe,

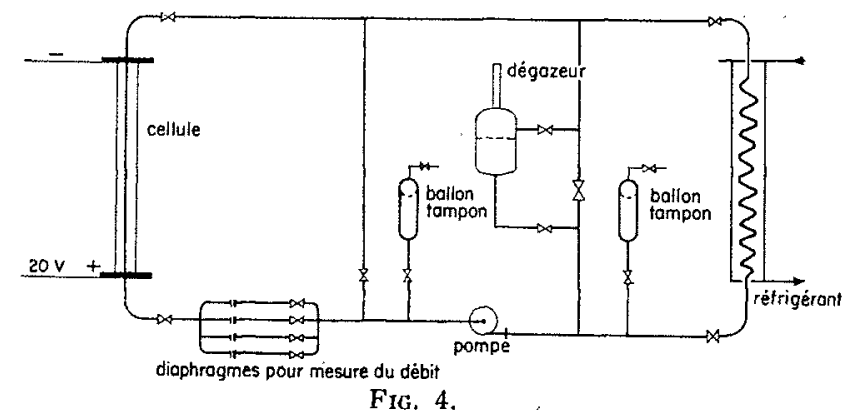

ballons-tampons, dégazeur, réfrigérant. L'alimentation est faite en courant continu $20 \mathrm{~V}$, $\dot{a}$ l'aide d'une batterie de redresseurs branchée par l'intermédiaire d'un transformateur sur un turbo-alternateur. Le réglage de puissance est commandé à partir de l'installation d'essais. La seule charge du turbo-alternateur est constituée par le chauffage du tube expérimental de facon à avoir un fonctionnement aussi stable que pos- sible. Toutes les tuyauteries sont en acier inoxydable pour éviter la corrosion. Le remplissage est fait en eau distillée. L'installation a été largement dimensionnée de telle sorte que nous

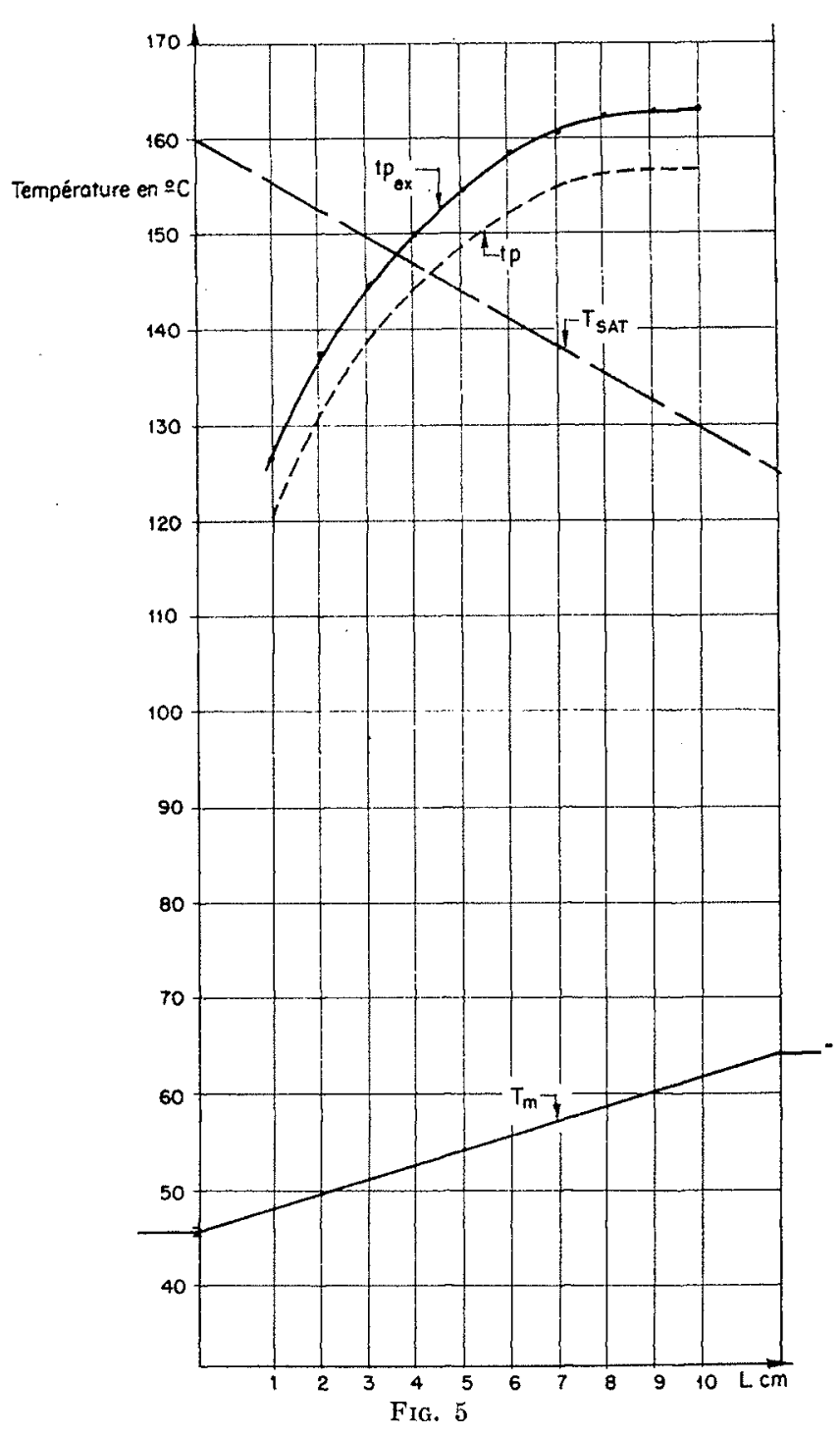

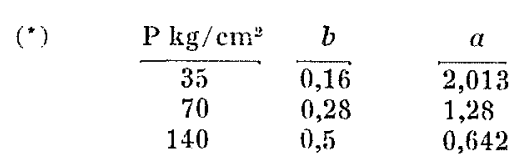




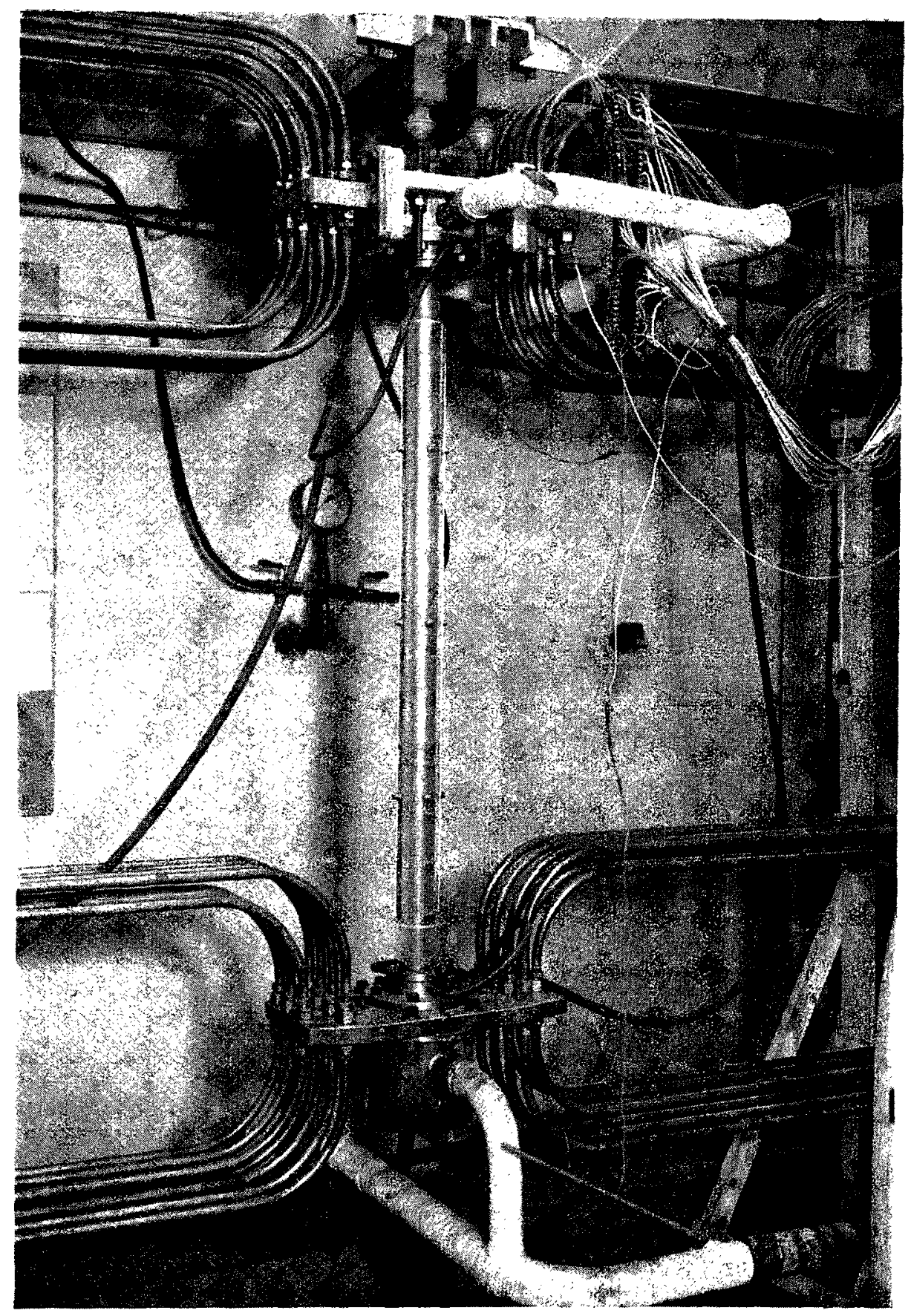

Cellule d'essai EL.3. Ensemble de la cellule d'essais au in stade.

avons pu monter jusqu'à une densité de $326 \mathrm{w} / \mathrm{cm}^{2}$, soit $2,82 \times 10^{6} \mathrm{kcal} / \mathrm{h} / \mathrm{m}^{2}$. Les températures de surface du tube ont été mesurées à l'aide de thermo-couples appliqués contre la paroi du tube chauffé soit à l'intérieur, soit à l'extéricur, suivant que le tube est refroidi extérieurement ou intérieurement Isa photographie montre l'installation.

Nous remarquerons qu'opérant constamment avec de l'eau distillée, nous n'avons pas eu de formation de dépôt ou d'oxydation gênantes pour les essais.

Nous commencerons par examiner les résultats des essais réalisés sur le tube refroidi par circulation d'eau intérieure, puisque ce sont toujours ces essais qui servent de base de comparaison. Dix thermo-couples ont été disposés à intervalles réguliers le long du tube expéri- 

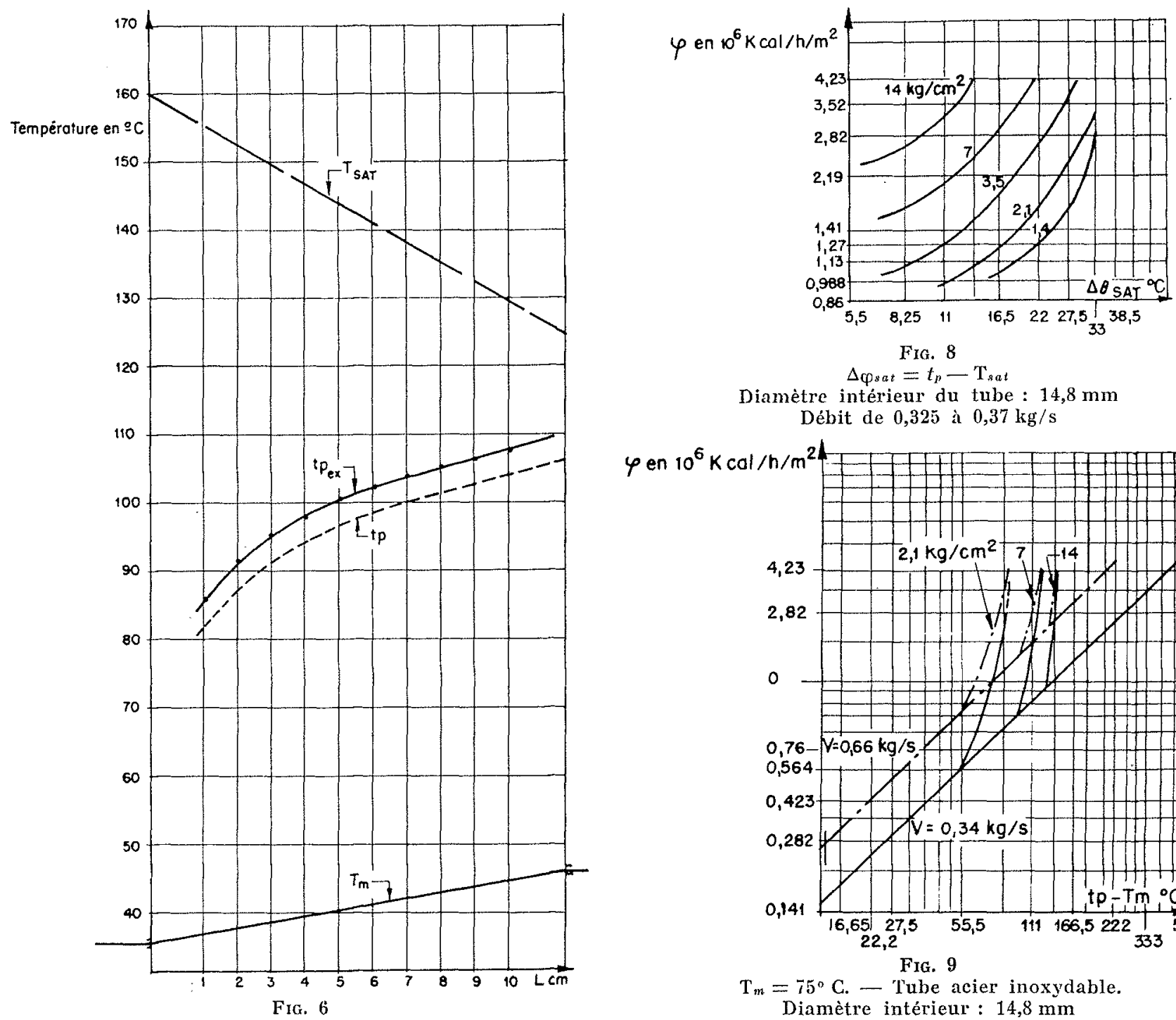

Fra. 8

$\Delta_{\varphi s a t}=t_{p}-\mathrm{T}_{s a t}$

Diamètre intérieur du tube : $14,8 \mathrm{~mm}$ Débit de 0,325 à $0,37 \mathrm{~kg} / \mathrm{s}$

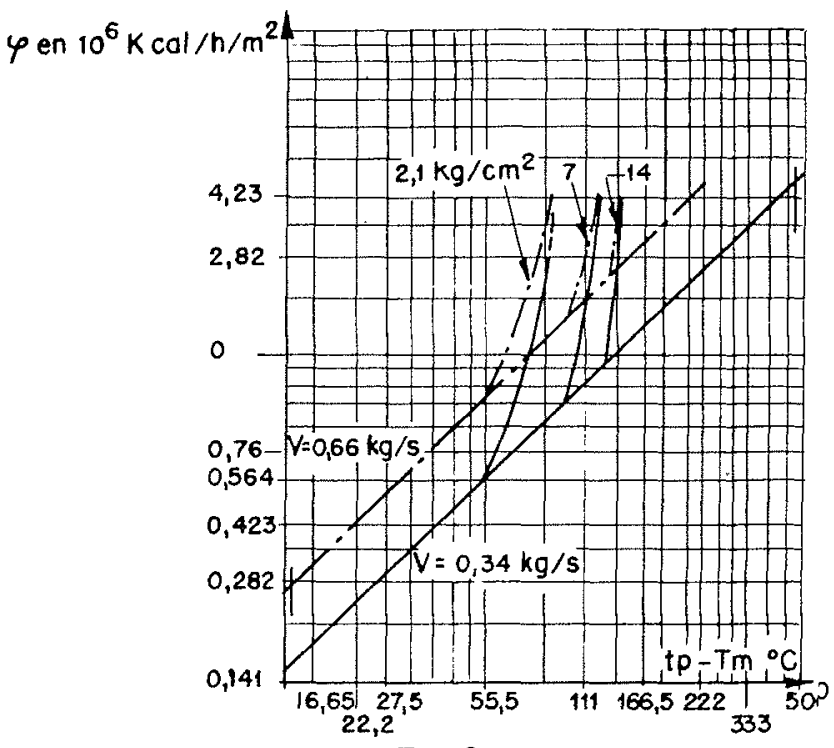

FIG. 9

$\mathrm{T}_{m}=75^{\circ} \mathrm{C}$. Tube acier inoxydable. Diamètre intérieur : $14,8 \mathrm{~mm}$

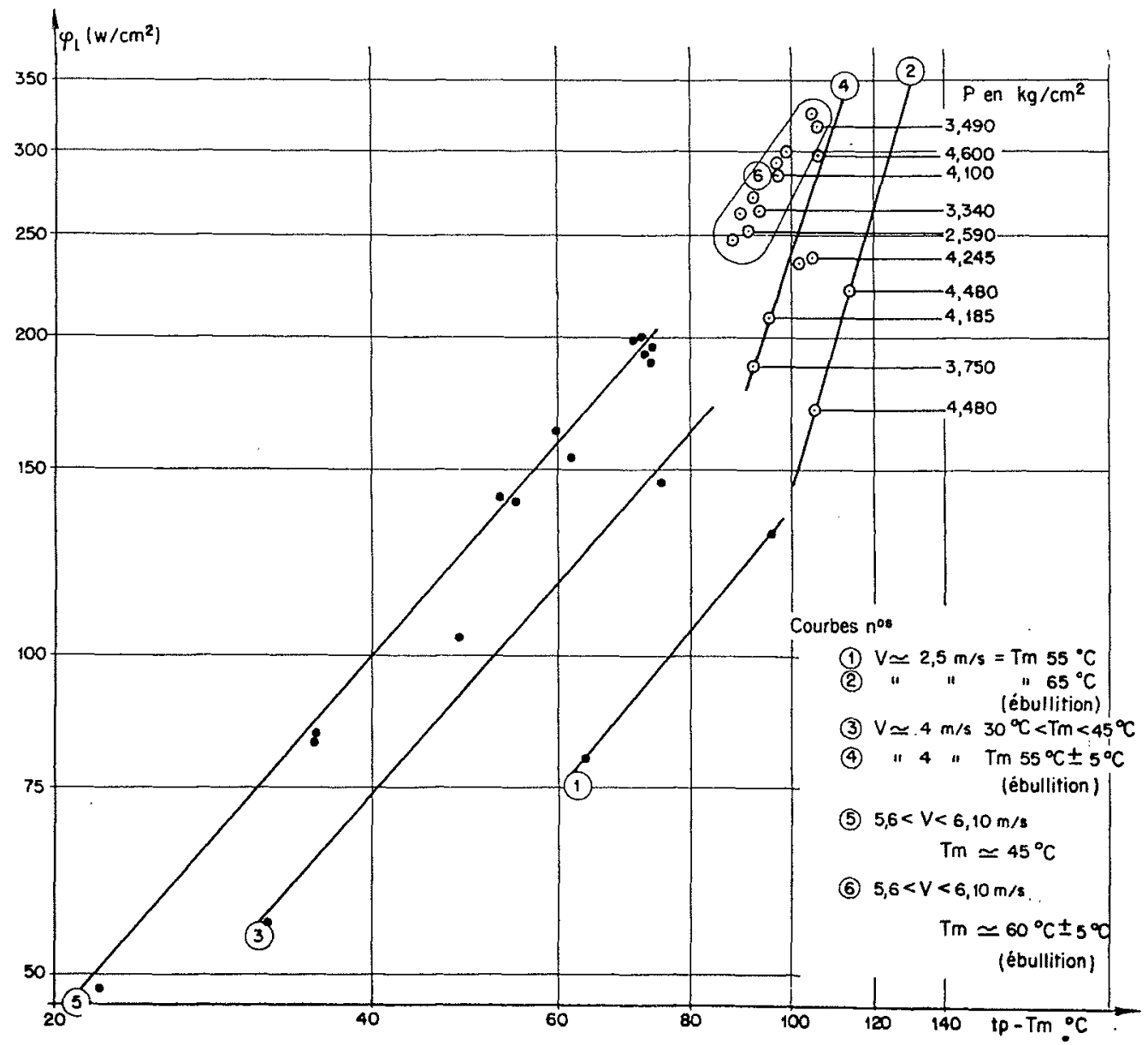

FIG. 7 


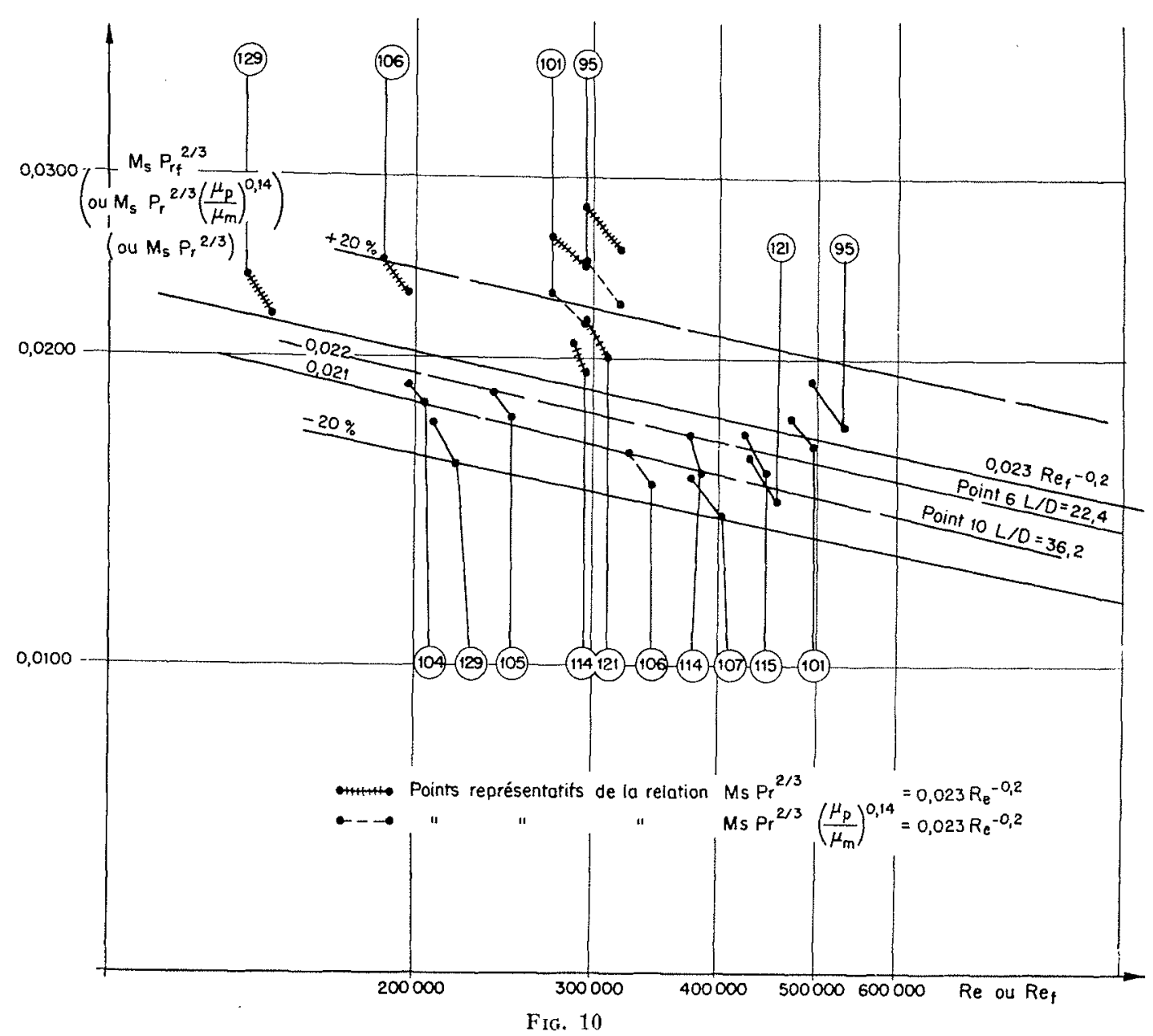

mental de diamètre 31 , épaisseur $1 \mathrm{~mm}$, longueur $1,220 \mathrm{~m}$.

La figure 5 donne un exemple des variations de température mesurées le long du tube, lors l'un essai où, dans la portion finale du tube, a lieu l'ébullition de surface. La différence est très nette par rapport à un graphique (fig. 6) correspondant à la convection forcée, sans ébullition. La figure 7 donne la représentation en diagramme logarithmique de la variation de la densité de flux de chaleur en fonction de $t_{p}-\mathrm{T}_{m}$. Les courbes sont tracées à vitesse constante pour une température de masse donnée.

La zone d'ébullition de surface avec bulles se distingue aisément de la zone sans ébullition.

L'influence de la pression sur l'ébullition de surface avec bulle n'a pas été étudiée. La courbe 6 de la figure 7 donne des résultats en opposition avec ceux des figures 8 et 9 , où la pression est déterminante $[5]$. Cette zone demande à être précisée par des essais ultérieurs.

Pour compléter cette représentation dans le cas de la convection forcée sans ébullition de surface, la figure 10 indique l'écart existant entre les résultats d'essais et la formule de Col- burn. A titre de comparaison, quelques points ont été calculés avec la formule de Colburn où $y, c_{p}, \lambda$ sont pris à la température de la masse du' fluide et avec celle comportant le lacteur de correction $\left(\mu_{p} / \mu_{m}\right)^{0,14}$; la dispersion plus grande obtenue justific le choix d'une température de référence pour ces caractéristiques physiques intermédiaires entre la température de

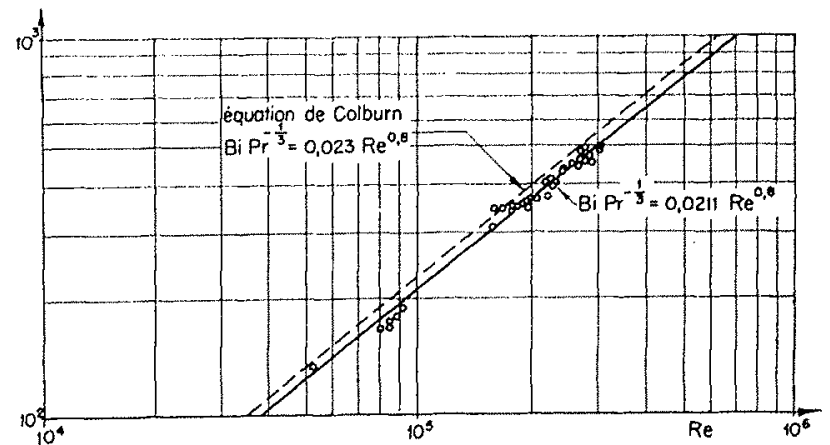

FIG. 11

Comparaison de résultats du Massaclussets Institute of Technology, avec l'équation de Colburn pour la section correspondant au rapport $\mathrm{J} / \mathrm{D}$ de 18

Resultats d'essai sans ébullition : $\mathrm{P}=105$ et $140 \mathrm{~kg} / \mathrm{cm}^{2}$ 
paroi et la température de la masse du liquide, la température retenue a été $\mathrm{T}_{f}=\left(l_{n}-1-\mathrm{T}_{m}\right) / 2$ par raison de simplicité.

Les points reliés par des lirets correspondent le premier au point où le régime d'ćcoulement turbulent est bien établi et le deuxième au dernier point de mesure. Si celui-ci s'écarte de la formule habituelle plus que le premier, nous pensons qu'il faut en rechercher la cause dans le fait que le rapport $L / D$ continuc à jouer un ròle minime mais non nésligeable; tant qu'il n'a pas atteint une certaine valeur limite qui peut varier suivant les conditions régnant à l'amonl du tube, le coefficient de transmission continue à décroître lentement. Il est intéressant de constater que le même phénomène a été observé dans des essais américains (fig. 11 et 12) à $140 \mathrm{~kg} / \mathrm{cm}^{2}$. Les valeurs des rapports $\mathrm{L} / \mathrm{D}$ sur ces vues sont à peu près les mêmes que celles considérées dans nos essais. Tous les résultats de la figure 10

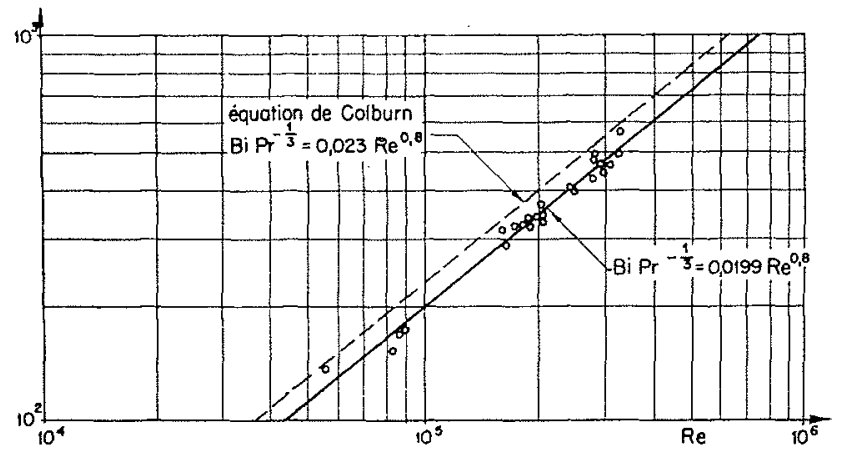

Fig. 12

Comparaison de résultats du Massachussels Institute of Technology, avec l'équation de Colburn pour la section correspondant au lapport $\mathrm{L} / \mathrm{D}$ de $4 !$. Résultats d'essai sans ébullition : $P=105$ et $140 \mathrm{~kg} / \mathrm{cm}^{2}$

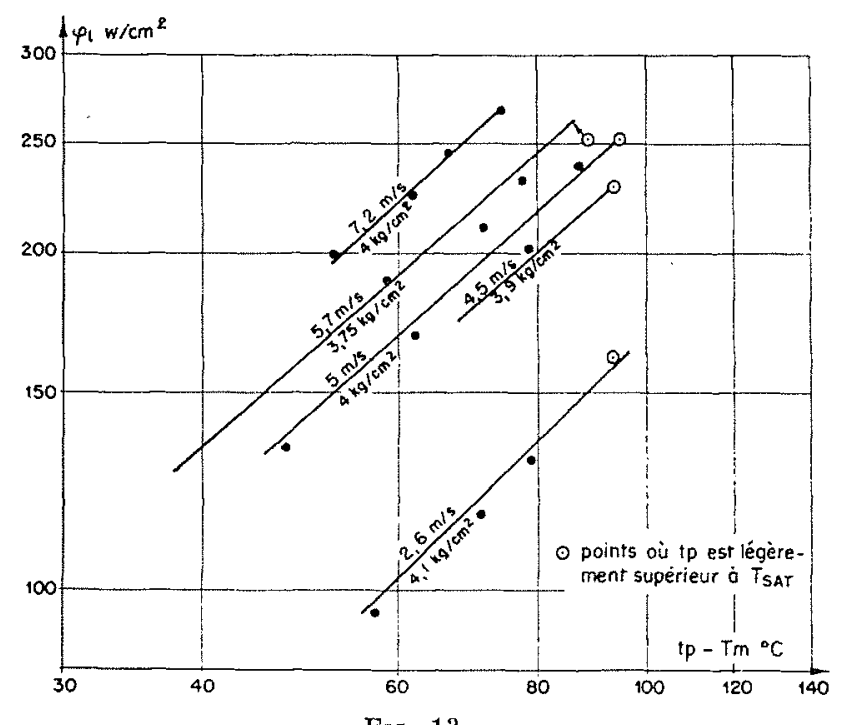

Fig. 13

Varialion de pore $t_{p} \ldots-\mathrm{T}_{2}$ $T_{m}$ constant $\overline{3} 6^{\circ} \mathrm{C}$

Réseau de courlues à vitesse constante. sans ébullition rentrent dans la limite de dispersion précisée par Mac Ansms t- $20 \%$.

Cependant, on peut proposer les relations suivantes:

$$
\begin{aligned}
& \text { pour }(\mathrm{L} / \mathrm{D})=22,4 \quad \operatorname{Ms~}_{\operatorname{Pr}_{f}^{2 / 3}}^{2 / 3}=0,022 \mathrm{Re}_{f}^{-0,2} \\
& \text { pour }(\mathrm{L} / \mathrm{D})=36,2 \mathrm{Ms} \mathrm{Pr}_{f}^{2 / 3}=0,021 \mathrm{Re}_{f}^{-0,2}\left(^{\star}\right)
\end{aligned}
$$

En conclusion, nos résultats en convection forcée sans ébullition sont en concordance avec les résultats connus de divers expérimentateurs.

Les expériences faites en espace annulaire: tube chanffant en aluminium de $29 \times 31$ mm, longueur $1.380 \mathrm{~mm}$, ayant même axe qu'un tube en plexiglass de $\varnothing 40 \mathrm{~mm}$ ont fait l'objet de graphiques analogues : figure 13 avec $\varphi=\mathrm{F}\left(t_{p}-\mathrm{T}_{m}\right)$, figures 14 et 15 qui montrent la formule de Colburn comme bonne en prenant pour diamètre équivalent la valeur $\mathrm{D}_{2}-\mathrm{D}_{1}$; la figure 16 donne la variation de en fonction de :

$$
t_{p}-\mathrm{T}_{\mathrm{s} a t} \mathrm{p}=a \Delta \Theta_{\mathrm{x} a t}{ }^{0,3 a} .
$$

La perte de charge, avec la même valeur de diamètre équivalent, est inférieure à celle prévue, mème si l'on fait la correction relative à un écoulement non isotherme (figs. 17).

Signalons un aspect qui montre l'intérêt présenté par ces essais; pour les mener à bien il n'a pas fallu moins de 6 tubes différents qui tous ont été détruits.

Ces premiers résultats, qui concernent un premier programme d'essais, sont encore modestes par rapport a ceux obtenus aux Etats-Unis; ils ont cependant permis de vérifier que le dispositif expérimental était bien rodé, et de répondre aux questions initiales posées pour la pile EL. 3.

De plus, ils donnent la possibilité d'orienter les travaux futurs en ce sens qu'ils nous ont fait apprécier l'importance relative des différents paramètres régissant le phénomène d'ébullition de surface avec bulles, et sentir les points qui doivent être approfondis. C'est ainsi qu'en particulier, l'influence de la pression de la teneur de l'eau en gaz dissous sont au premier rang de nos préoccupations, comme la variation dans le temps de l'état de surface dñ tube chauffé. D'autre part, certaines questions sont à éclaircir : en premier lieu, le retard apporté à l'amélioration du coefficient de transmission lorsque $t_{p}>\mathrm{T}_{\text {sat }}$, retard qui apparaît seulement dans certaines conditions mal définies; en second lieu, les phénomènes d'instabilité lorsque l'on s'approche de la densité du flux maximum, nous en avons eu un exemple

(*) (A moter que Mac Absus, dans son traté, donne des résultats pour aulant que $\mathrm{L} / \mathrm{D}$ ) $>60$ ). 


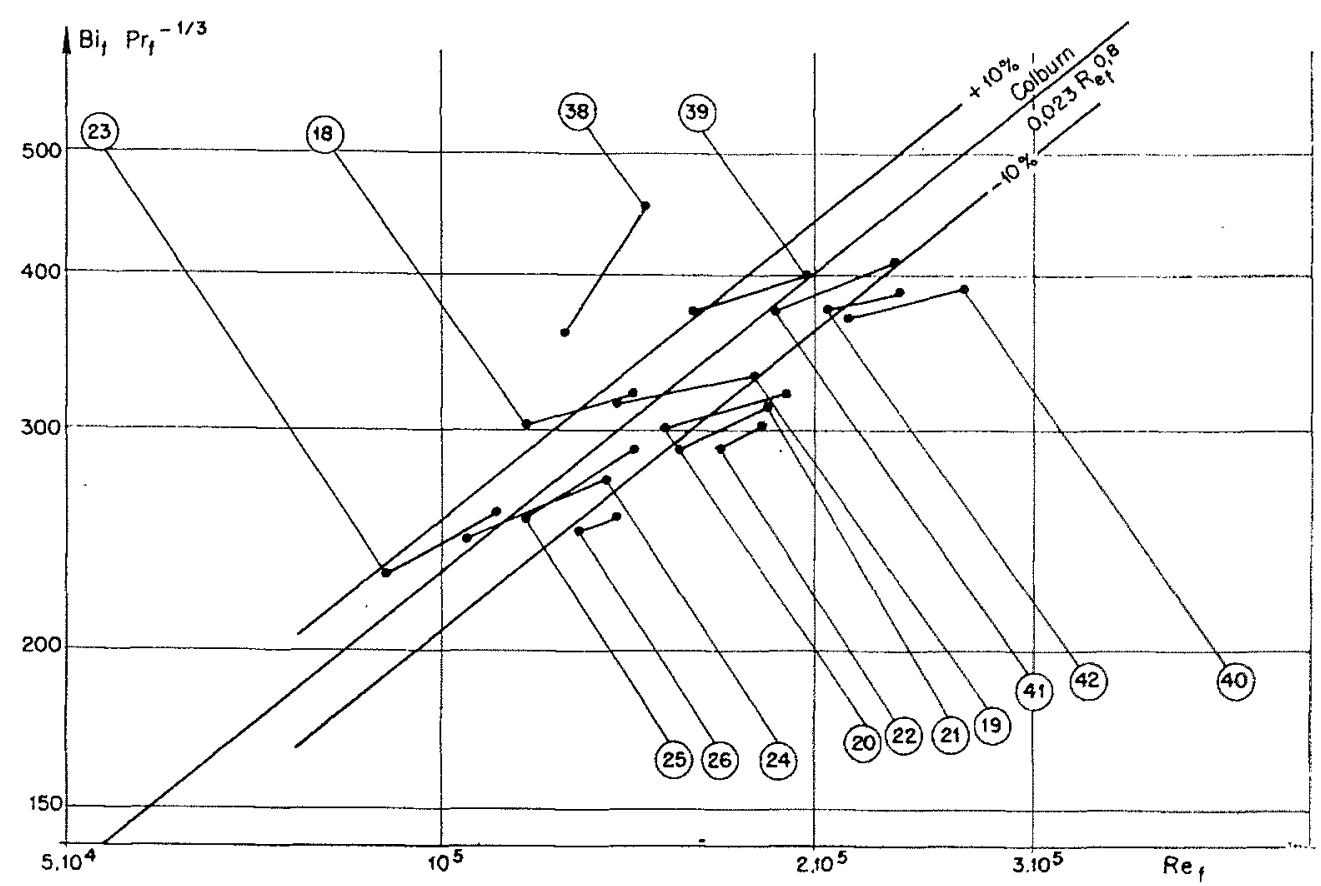

FIG. 14

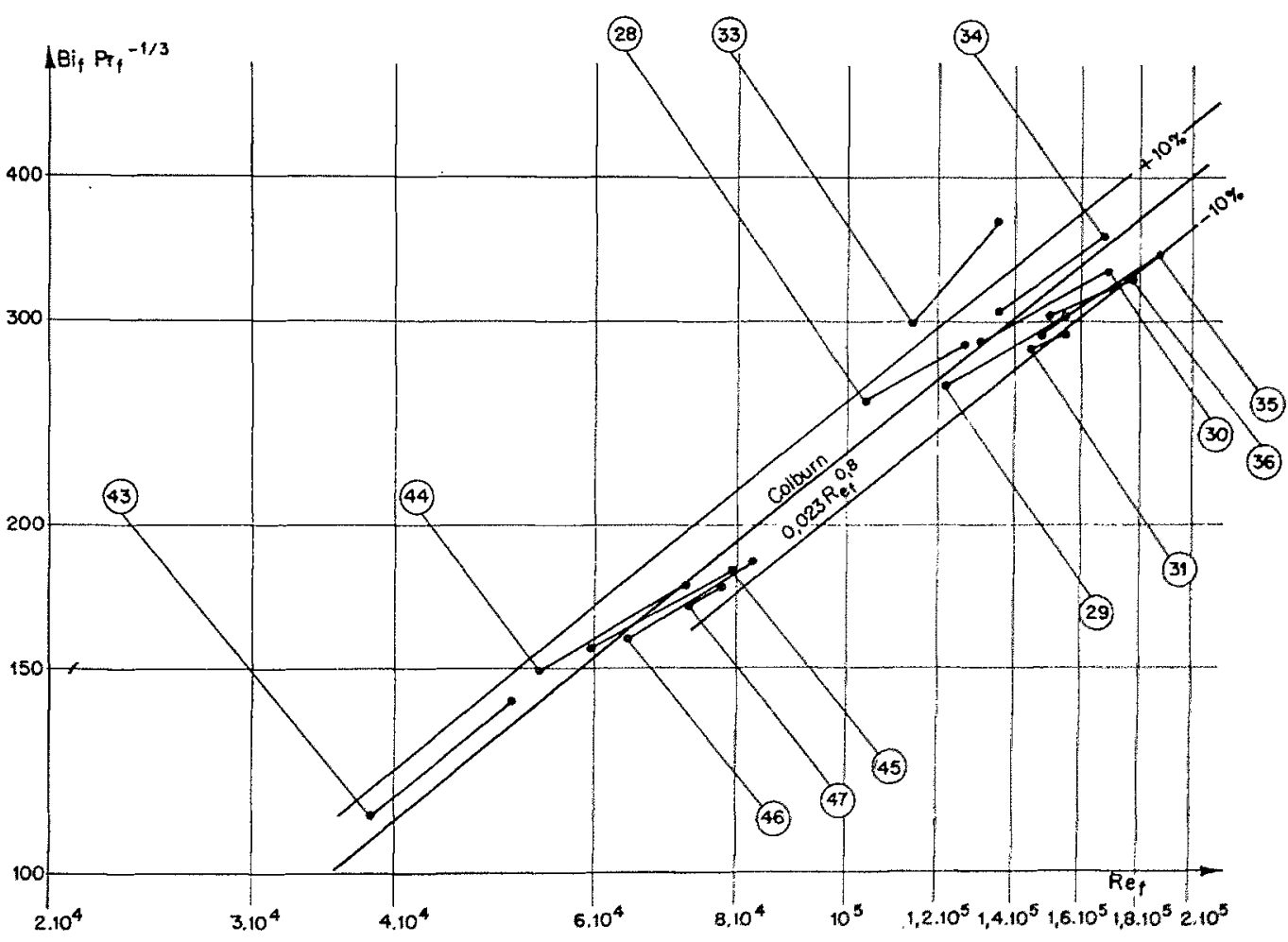

FIa. 15

en opérant à flux constant el en réduisant le débit jusqu'à une valeur pourtant admissible, une surchauffe locale s'est produile entrainant la destruction du tube.
Soulignons onlin, car cotle caractéristique est un émenent déterminam pour aboutiv au but fixé dans ce genre d'clude, l'esprit de collaboration dans lequel ces travaux ont ele faits aussi 


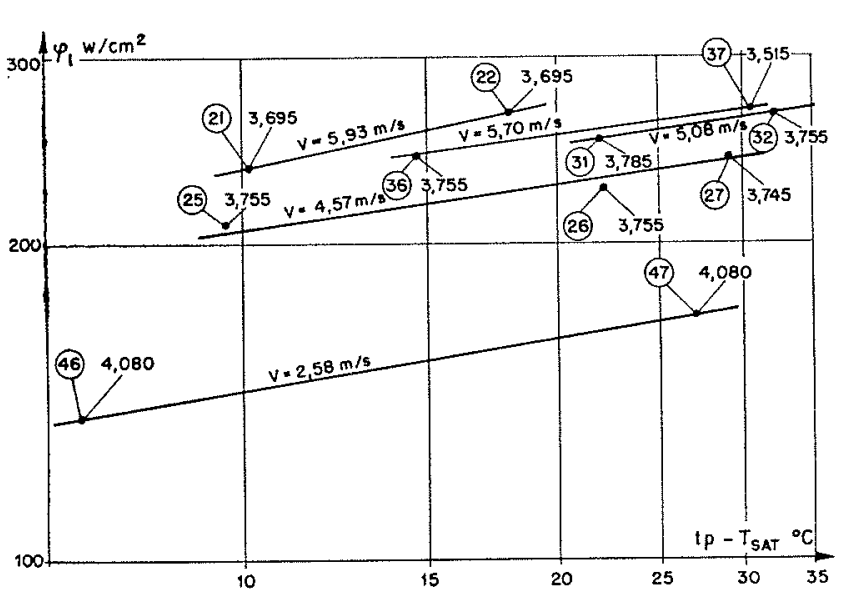

FIG. 16

bien avec le Commissariat à l'Énergie Atomic̨ue et les Chantiers de l'Atlantique qu'avec les divers techniciens mis à contribution, chacun dans

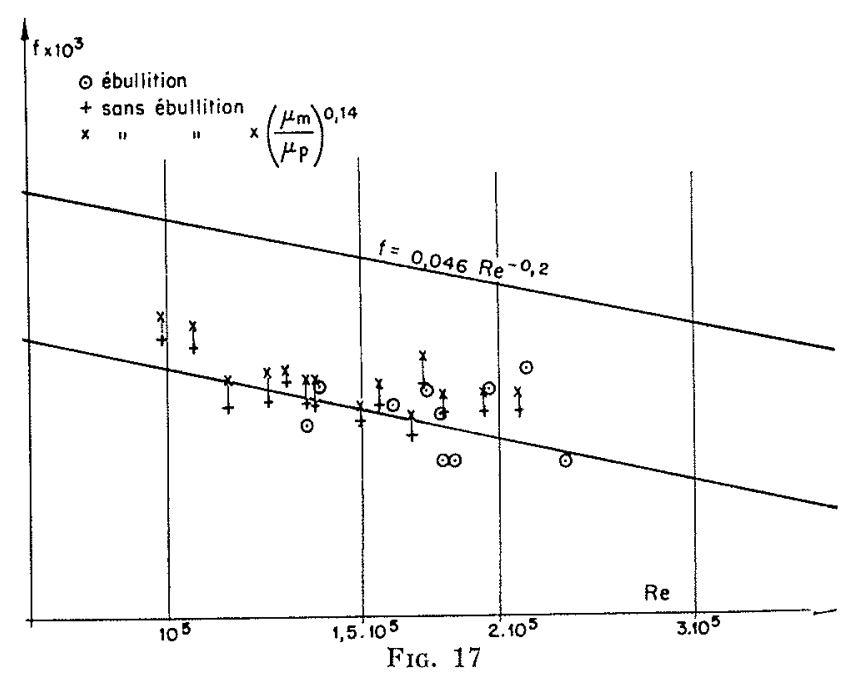

leur spécialité, pour l'étude, la construction et la mise au point de l'installation d'essais et l'interprétation des résultats.

\section{BIBLIOGRAPHIE}

(1) W. H. Mc Adams. - Heat transmission. $3^{\text {e édition }}$ 1954.

(2) Jens. - Mechanical Engineering. Décembre 1954.

(3) Gunther. - Trans. A.S.M.E. Février 1951.

(4) W.H. MC Adams, W.E. Kennell. - Industrial and engineering chemistry. Sept. 1949.
(5) Kreith et Summerfed. - Trans A.S.M.F. Octobie 1949.

(6) W.M. Rohsenow. - Trans. A.S.M.E. 1952.

(7) JeNs et LotTes. - Rapport A.E.C., Anl. 4627. Mai 1951.

(8) Jens et Leppert. - J. Amer, Soc. Nav. Engrs, 1955.

\section{I S C U S S I O N}

Président : M. Ginhat

Sur la demande de M. Beunt-Henka, M. Butzisach précise que les essais rapportés ont été faits sous une pression de 2 à $5 \mathrm{~kg}$.

M. le Président félicite vivement M. BuTzbach et indique que des essais du genre de ceux effectués sur la cellule à eau bouillante ront débuter dans un très proche avenir à l'échelle industrielle aux Etats-Unis, dès la mise en service de la centrale de Philo utilisant de l'eau à l'état super-critique: dans cette centrale, trois ou quatre cents points de mesures de température sont reliés à un téléscripteur qui les transmettra immédiatement à des machines à calculer installées à $400 \mathrm{~km}$ de la centrale et chargées du dépouillement. On peut donc espérer disposer d'ici quelques mois d'une masse importante de renseignements sur cette question. 